\title{
METABOLISMO RESPIRATÓRIO DO MOLUSCO ANFÍBIO Pomacea lineata (SPIX, 1827): INFLUÊNCIA DA CONDIÇÃO ALIMENTAR NO CONSUMO DE OXIGÊNIO DO ANIMAL TOTAL E DE SEU MÚSCULO RADULAR
}

\section{RESPIRATORY METABOLISM OF THE AMPHYBIOUS} MOLLUSC Pomacea lineata (SPIX, 1827): INFLUENCE OF ALIMENTARY CONDITION ON THE OXYGEN CONSUMPTION OF WHOLE ANIMAL AND ITS RADULAR MUSCLE.

\section{Mendonça, F. A. S. ${ }^{1}$ and Santos, G. M. T. ${ }^{1}$}

${ }^{1}$ Centro Universitário Hermínio Ometto - UNIARARAS - Araras - SP

\section{RESUMO}

A finalidade do presente trabalho foi estudar o metabolismo respiratório de Pomacea lineata, um gastrópode anfíbio, e determinar o consumo de oxigênio do animal inteiro e seu respectivo músculo radular em diferentes condições alimentares (jejunos e alimentados). Essa espécie é interessante no seu aspecto fisioecológico, pois trata-se de um molusco importante como bioindicador da qualidade de um ecossistema.

Verificou-se que o metabolismo respiratório, estimado em termos de variação da taxa respiratória, diminuiu significativamente $(\mathrm{P}<0,05)$ sob o efeito de jejum nos animais inteiros (0,473 nos alimentados e 0,317 nos jejunos), assim como nos respectivos músculos radulares $(0,884$ nos alimentados e 0,521 nos jejunos).

A taxa respiratória média dos músculos radulares foi significativamente maior $(\mathrm{P}<0,05)$, que a taxa respiratória média dos animais inteiros (alimentados e jejunos) devido à grande quantidade de mioglobina presente nesses músculos.

Os resultados obtidos estão em conformidade com as características morfofuncionais de $P$. lineata. Seu músculo radular é usado ativamente na obtenção do 
alimento e faz parte do órgão raspador do animal. Esse músculo necessita de grandes quantidades de oxigênio que o animal retira da água ou do meio aéreo, justificando a posição destacada desse gastrópodo anfíbio como bioindicador da qualidade de um ecossistema.

Palavras-Chave: Pomacea lineata, molusco, gastrópoda, taxa respiratória, condição alimentar.

\section{ABSTRACT}

The present work aimed to investigate the respiratory metabolism of the amphibious gastropoda Pomacea lineata, and to measure the oxygen-consumption of both whole animal and its radular muscle in different alimentary conditions (whether fed or starved). The species is interesting in a physioecologic aspect, because it is an important snail as a bioindicator of ecosystem quality .

It was observed that respiratory metabolism obtained from respiratory rate variation was significantly reduced $(\mathrm{P}<0,05)$ under starvation effect in the whole animals $(0,473$ in whether fed and 0,317 in starved), as well as in the respective radular muscles $(0,884$ in whether fed and 0,521 in starved).

The respiratory rate average of the radular muscles was significantly greater $(\mathrm{P}<0,05)$ than respiratory rate average of the whole animals (whether fed and starved) as a result of the myoglobin quantity from these muscles.

These results are in accordance with the morphofunctional characteristics of $P$. lineata. Its radular muscle works out actively for getting feed and constitutes the animal scraping bucal organ. This muscle needs enormous quantity of oxygen obtained from water or air environment, in order to justify the important role played for these amphibious gastropoda as a bioindicator of ecosystem quality.

Key-words: Pomacea lineata, mollusc, gastropoda, respiratory rate, alimentary condition.

\section{INTRODUÇÃO}

O interesse no estudo dos moluscos se deve, tal como no caso de muitos outros animais, à sua participação na manutenção do equilíbrio ecológico da natureza, intervindo na reciclagem de detritos, ainda que alguns possam eventualmente representar uma praga para a agricultura (DEYRUP-OLSEN \& MARTIN, 1987). Destaca-se ainda a importância desses animais como bioindicadores da qualidade de um ecossistema. 
Segundo Marques e Barbosa (2001), moluscos estão entre os macroinvertebrados bioindicadores uma vez que são sensíveis à poluição e variações do habitat. Nos anos 60 surgiram as primeiras tentativas de quantificar o grau de poluição levando em conta a incidência de uma determinada espécie e sua tolerância à poluição.

Melo (2000) desenvolveu uma metodologia para verificação de toxidade em mananciais de água doce no nordeste do Brasil, visando uma complementação nos métodos convencionais de análise de água. Para tal, utilizou como bioindicador, Pomacea lineata, cujos filhotes apresentaram uma elevada sensibilidade ao pesticida "Paraquat" sendo possível observar uma nítida resposta do tipo "dose-dependente".

Esses dados justificam a necessidade de se estudar moluscos, sobretudo as espécies anfíbias como $P$. lineata, para melhor compreender as suas relações fisiológicas com o habitat e, então, determinar a sua importância no biomonitoramento. Além disso, a variabilidade dos moluscos torna atraente a investigação de como estes animais intactos, ou porções isoladas dos mesmos, se comportam face à variações osmóticas ou iônicas do meio (CRAVEIRO, 1997).

Os moluscos gastrópodes empregam diversos mecanismos de alimentação (ANKEL, 1938; GRAHAM, 1955), tais como, raspagem, perfuração ou filtração. O mais importante fator que possibilita esta diversificação de mecanismos é o complexo rádula/bucal (OWEN, 1966).

Em Pomacea, a fonte de alimentação consiste de angiorpermas aquáticas.Este alimento, usualmente uma folha, é mantido pelo pé e agarrado pelos dentes centrais da rádula protudida. Esta protusão, bem como a retração da rádula, é operada por músculos radulares bastante peculiares. Tais músculos não são estriados e, em invertebrados, este tipo de músculo é geralmente constituído de estruturas bem delimitadas e excisáveis, íntegras, para o trabalho experimental. Em 1872, LANKESTER (FÄNGE \& MATTISSON, 1958 b), considerou que o pigmento vermelho desses músculos é a hemoglobina o que foi confirmado por Mendel e Bradley (1905). A propósito, Manwell (1963), informou que o teor deste pigmento nos músculos radulares de moluscos marinhos, que ele chamou de mioglobina, é de 3 a 4\%. Esta mioglobina tem uma maior afinidade pelo oxigênio que a hemocianina destes moluscos, o que sugere que participa do transporte do gás da hemolinfa para o tecido.

A presença de mioglobina em músculos estriados de vertebrados tem sido interpretada como um meio de armazenamento de oxigênio para enfrentar a grande demanda deste gás no funcionamento muscular e, também, para facilitar sua entrada no músculo, embora não esteja ainda claro como poderia contribuir neste último caso (WITTEMBERG, 1970). O consumo de oxigênio do músculo radular de moluscos é, reconhecidamente, elevado (FÄNGE E MATTISSON, 1958 b). Assim, a hemoglobina do músculo radular pode, presumivelmente, agir durante curto período de tempo como 
armazenador de oxigênio.

De acordo com Silva et al. (2001), o estudo do metabolismo respiratório é fundamental para a compreensão dos aspectos básicos da biologia, fisiologia, e comportamento metabólico dos animais, principalmente quando é relacionado com o tamanho, sexo, condição alimentar e outros fatores intrínsecos e extrínsecos. Com relação a alguns destes parâmetros o metabolismo respiratório de $P$. lineata já foi estudado por Santos e Mendes (1981) e Santos et al. (1987) em relação ao tamanho corpóreo, sexo, desenvolvimento, temperatura e tensão de oxigênio.

O presente trabalho teve como meta investigar o metabolismo respiratório de $P$. lineata e seu respectivo músculo radular isolado e imerso em solução salina, em diferentes condições experimentais, utilizando-se de animais jejunos e alimentados.

\section{MATERIAL E MÉTODOS}

Os espécimes de Pomacea lineata foram coletados em várias localidades situadas nos municípios de Rio Claro, Cordeirópolis e Pirassununga e transportados para tanques do laboratório de Zoologia da UNESP - Rio Claro e mantidos em condições comparáveis às de seu habitat natural, onde permaneceram até a hora dos experimentos.

Para as medidas do consumo horário de oxigênio $\left(\mu 1 \mathrm{O}_{2} / \mathrm{h}\right)$, necessárias ao cálculo da taxa respiratória $(\mu \mathrm{l} / \mathrm{mg}(\mathrm{s}) / \mathrm{h})$, foi usado o respirômetro de Warburg, de banho termostatizado e regulado para $25^{\circ} \mathrm{C}$.

Primeiramente foram feitas medidas do consumo de $\mathrm{O}_{2}$ do animal total usando-se frascos de Warburg com capacidade média de $130 \mathrm{ml}$. No bulbo lateral foi colocado $1 \mathrm{ml}$ de $\mathrm{KOH} 14 \%$ para absorção do $\mathrm{CO}_{2}$ eliminado durante o processo respiratório. Em cada frasco foram também colocados $35 \mathrm{ml}$ de água na qual ficavam imersos os animais.

Os músculos radulares de $P$. lineata foram colocados em frascos de Warburg do tipo padrão, com capacidade média de $5 \mathrm{ml}$, providos de bulbo lateral e poço central onde foi usado $0,05 \mathrm{ml}$ de $\mathrm{KOH}$ a $14 \%$ para absorção de $\mathrm{CO}_{2}$. O músculo foi suspenso em $1 \mathrm{ml}$ de salina de Little com a seguinte composição em g/1000ml: $\mathrm{NaCl}=2,38, \mathrm{KCl}$ $=0,16, \mathrm{CaCl} 2=1,40, \mathrm{NaHCO} 3=0,50$ (LITTLE, 1968). Os animais inteiros não foram submetidos durante os experimentos a nenhuma agitação externa do sistema, mas os músculos radulares foram submetidos a 120 oscilações/minuto.

Foram utilizados seis frascos por experimento, sendo que um deles serviu 
como termobarômetro (sem animal ou músculo). Os conjuntos foram levados ao banho com as torneiras do manômetro abertas. Após 15 minutos de espera para a termoestabilização do sistema, os níveis dos manômetros foram reajustados, as torneiras foram fechadas e, imediatamente, tomadas as leituras iniciais. As leituras seguintes fizeram-se em intervalos de 15 minutos, durante 75 minutos. A obtenção dos volumes (em $\mathrm{ml}$ ), dos animais e dos músculos radulares, fez-se por deslocamento do nível de líquido em proveta graduada (água, no caso de animais inteiros, ou salina de Little, para os músculos radulares), onde o material foi introduzido. Ao término deste período os animais inteiros (sem a concha) e os músculos radulares foram colocados em estufa a $110^{\circ} \mathrm{C}$ por 24 horas para obtenção do peso seco (S) usado no cálculo da taxa respiratória. Os seguintes procedimentos experimentais foram seguidos:

1.Animais alimentados;

2.Seus respectivos músculos radulares;

3.Animais jejunos (24 horas);

4.Seus respectivos músculos radulares.

\section{RESULTADOS E DISCUSSÃO}

Os resultados obtidos para Pomacea lineata constam da Tabela I - animais alimentados e seus respectivos músculos radulares. $(\mathrm{n}=25)$ e da Tabela II - animais jejunos e seus respectivos músculos radulares $(n=25)$. Os animais estão ordenados em ordem crescente de pesos.

A análise dos resultados indicou que:

a) não houve significância nas correlações entre peso seco do animal inteiro (sem a concha) e peso seco de seus respectivos músculos radulares tanto nos alimentados $(\mathrm{r}=$ 0,34; $\mathrm{P}>0,05)$, como nos jejunos $(\mathrm{r}=0,37 ; \mathrm{P}>0,05)$.

b) a massa muscular radular representou, em média, $1,57 \%$ da massa do animal inteiro para os alimentados (Tabela I e Figura 1) e 2,01\% para os jejunos (Tabela II e Figura 2).

c) a diferença entre as percentagens médias do conjunto da massa muscular em relação ao peso corpóreo foi significativamente maior $(\mathrm{P}<0,05)$ nos animais jejunos $(2,01 \% \mathrm{X}$ $1,57 \% ; \mathrm{t}=3,73>\mathrm{tc}=2,01$ ). Isto se deve, provavelmente, ao fato de ser o músculo constituído basicamente de proteína, que não é utilizada como fonte imediata para obtenção de energia durante o jejum, a não ser que este seja prolongado, o que não ocorreu nos experimentos em questão (jejum de 24 horas ). 
d) não houve correlação significativa entre consumo horário de oxigênio de animais inteiros e consumo horário de oxigênio de seus respectivos músculos radulares, tanto entre os alimentados $(r=0,097 ; \mathrm{P}>0,05-$ Tabela $\mathrm{I})$ como entre os jejunos $(\mathrm{r}=0,24 ; \mathrm{P}>$ 0,05 - Tabela II).

Entretanto, a percentagem média do consumo horário de oxigênio dos músculos radulares, em relação ao consumo horário de oxigênio dos animais inteiros, não apresentou diferença significativa quando comparamos jejunos (Tabela II e Figura 4) e alimentados (Tabela I e Figura 3) respectivamente. $(3,43 \%$ x 3,33\%; $t=0,16<\mathrm{tc}=2,03)$.

A literatura é escassa sobre relações entre peso corpóreo e consumo de oxigênio em animais inteiros e de órgãos isolados em moluscos. Churchwell (1973) verificou que no bivalve Haliotis cracherodii, a respiração do animal inteiro representou de 27 a $58 \%$ do máximo da somatória da respiração tecidual. Não há dados percentuais sobre a respiração de cada órgão.

Em diferentes crustáceos decápodes braquiuros, Vernberg (1956) verificou, que em comparação com os valores de $\mathrm{VO}_{2}$ (animal inteiro), as taxas respiratórias do tecido branquial ficaram entre $2,35 \%$ (Ocypode) e $0,42 \%$ (Libinia) e que as oscilações refletiriam adaptações fisiológicas a regiões, desde níveis inferiores ao de maré vasante, até a terra firme. Assim, a mais alta percentagem estaria na espécie terrestre e extremamente ativa (Ocypode) e a menor percentagem na espécie marinha e relativamente lenta (Libinia).

$\mathrm{O}$ valor do $\mathrm{VO}_{2}$ encontrado para o músculo radular de $P$. lineata em relação ao animal inteiro $(3,33 \%)$ é relativamente alto. Trata-se de um músculo bastante ativo, que necessita de um grande suprimento de oxigênio. Assim, a mioglobina do músculo radular pode, presumivelmente, agir durante um tempo como um armazenador de oxigênio.

e) tanto a taxa respiratória dos animais inteiros $\left(0,473 \mu \mathrm{lO}_{2} / \mathrm{g}(\mathrm{s}) / \mathrm{h}\right.$ nos alimentados e 0,317 nos jejunos), como a taxa respiratória dos seus músculos radulares $\left(0,884 \mu \mathrm{lO}_{2} / \mathrm{g}\right.$ $(\mathrm{s}) / \mathrm{h}$ nos alimentados e 0,521 nos jejunos) diminuíram, significativamente $(\mathrm{P}<0,05)$, quando se comparou alimentados com jejunos $(\mathrm{t}=2,28>\mathrm{tc}=2,014$ : animais inteiros; $\mathrm{t}$ $=5,55>\mathrm{tc}=2.01:$ músculos radulares).

Por outro lado, os dados da Tabela I e II também mostram, que tanto em alimentados como em jejunos, houve correlações significativas entre:

- peso do animal e sua taxa respiratória ( $\mathrm{r}=0,50$ nos alimentados e $\mathrm{r}=0,71$ nos jejunos $; \mathrm{P}<0,05)$ 
- peso dos músculos radulares e sua taxa respiratória $(\mathrm{r}=0,41$ nos alimentados e $r=0,49$ nos jejunos; $\mathrm{P}<0,05$ )

O metabolismo respiratório, estimado em termos de variação de taxa respiratória, quase sempre altera-se sob efeito do jejum em outros animais. Uma diminuição da taxa respiratória foi observada em diplópodos por BYZOVA (1967), GROMYSZ-KALKOWSKA (1970), GROMYSZ-KALKOWSKA e STOJALOWSKA (1971), GROMYSZ-KALKOWSKA (1973) e SILVA Et Al. (2001). Este fato também foi observado por Ditadi et al. (1997) no hemicordato Glossobalanus crozieri. Em P. lineata, os resultados obtidos com animais jejunos confirmam estas observações, tanto no que se refere ao animal inteiro como em seu músculo radular.

f) a taxa respiratória média dos músculos radulares foi significativamente maior, ( $\mathrm{P}<$ $0,05)$, que a taxa respiratória média dos animais inteiros (alimentados e jejunos), ou seja, em termos de miligramas de peso seco, os animais inteiros respiraram menos que seus músculos radulares.

Estudos com respiração do músculo radular do molusco Buccinum undatum (FÄNGE \& MATTISSON, 1958) mostraram alta taxa respiratória $\left(0,620 \mu \mathrm{IO}_{2} / \mathrm{g}(\mathrm{s}) / \mathrm{h}\right)$ explicada pela abundância de mitocôndrias nas fibras musculares e presença de mioglobina nas mesmas. Também o músculo radular de $P$. lineata apresentou um alto valor da taxa respiratória e, novamente, pode-se inferir que em sua constituição química encontra-se grande quantidade de mioglobina, o que será estudado futuramente.

Os resultados obtidos estão em conformidade com as características morfofuncionais de $P$. lineata. Seu músculo radular é usado ativamente na obtenção do alimento e faz parte do órgão raspador do animal. Esse músculo necessita de grandes quantidades de oxigênio, que o animal retira da água ou do meio aéreo, justificando a posição destacada desse gastrópodo anfíbio como bio-indicador da qualidade de um ecossistema.

TABELA I - Peso seco, consumo horário de $\mathrm{O}_{2}$ e taxa respiratória de Pomacea lineata (animal inteiro alimentado e músculo radular inteiro). Animal inteiro imerso em água; músculo radular imerso em solução salina de Little. Temperatura $25^{\circ} \mathrm{C}$.

\begin{tabular}{|c|c|c|c|c|}
\hline $\begin{array}{l}\text { Peso } \\
\text { seco } \\
(\mathrm{mg})\end{array}$ & $\begin{array}{l}\text { Peso } \\
\text { músculo/animal } \\
\text { inteiro }\end{array}$ & $\mu \mathrm{I} \mathbf{O}_{2} / \mathbf{h}$ & $\mid \begin{array}{l}\mathrm{VO} \\
\text { músculo/VO } \\
\text { animal }\end{array}$ & $\begin{array}{c}\mu \mathrm{l} \mathrm{O} \mathbf{O}_{2} / \mathbf{m g} \\
(\mathbf{s}) / \mathbf{h}\end{array}$ \\
\hline
\end{tabular}




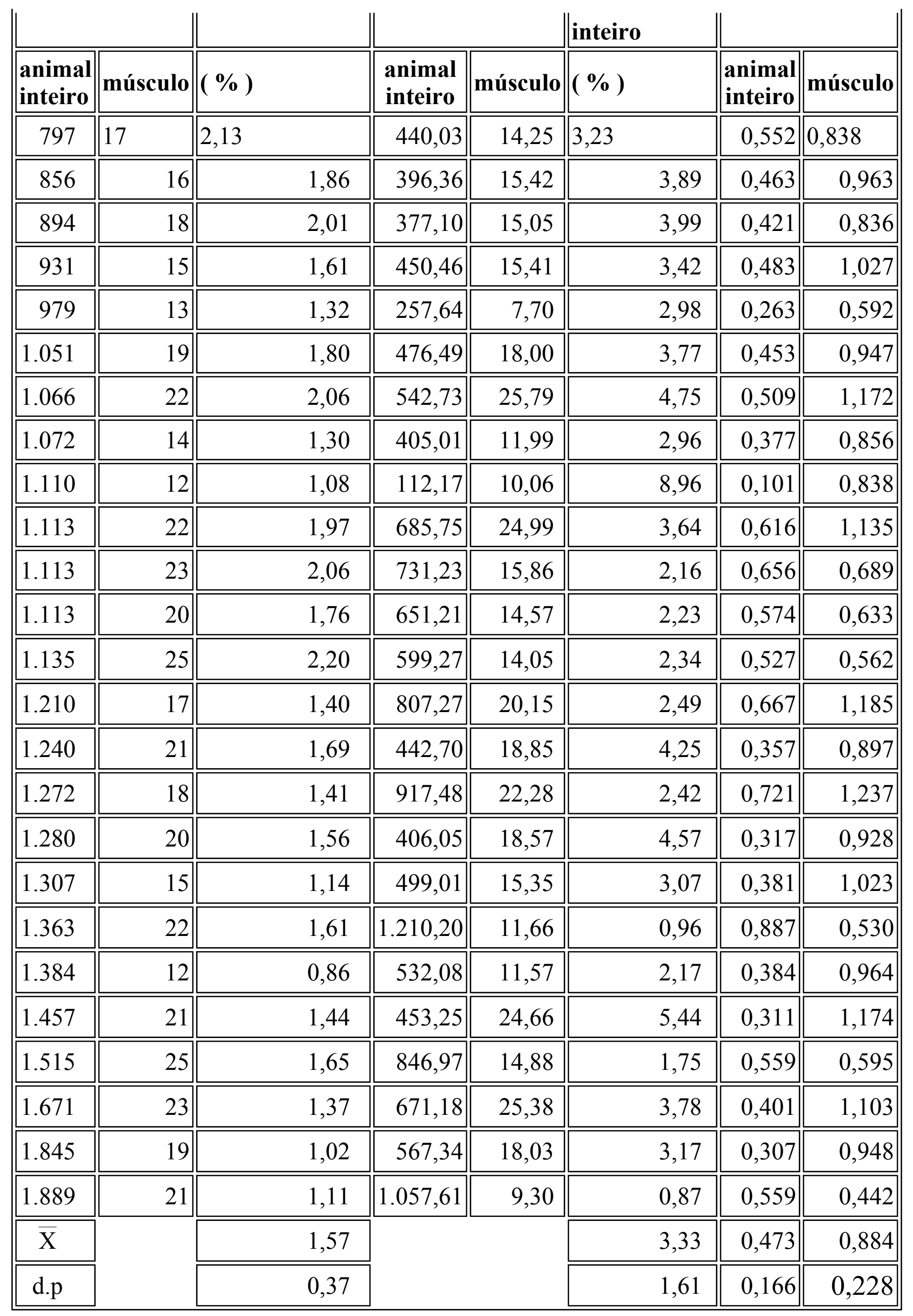


Tabela II - Peso seco, consumo horário de $\mathrm{O}_{2}$ e taxa respiratória de Pomacea lineata (animal inteiro jejuno de $24 \mathrm{~h}$ e músculo radular inteiro). Animal inteiro imerso em água; músculo radular imerso em solução salina de Little. Temperatura $25^{\circ} \mathrm{C}$.

\begin{tabular}{|c|c|c|c|c|c|c|c|}
\hline $\begin{array}{r}\text { Peso } \\
\text { (m) }\end{array}$ & $\begin{array}{l}\text { seco } \\
\text { g) }\end{array}$ & $\begin{array}{l}\text { Peso } \\
\text { músculo/animal } \\
\text { inteiro }\end{array}$ & $\mu \mathbf{I O}$ & $2 / h$ & $\begin{array}{c}\mathrm{VO}_{2} \\
\text { músculo/VO } \\
\text { animal }\end{array}$ & $\mu \mathbf{l} \mathbf{O}_{2}$ & $\begin{array}{l}\text { mg (s) / } \\
\mathbf{h}\end{array}$ \\
\hline $\begin{array}{l}\text { animal } \\
\text { inteiro }\end{array}$ & músculo & $(\%)$ & $\begin{array}{l}\text { animal } \\
\text { inteiro }\end{array}$ & músculo & $(\%)$ & \begin{tabular}{|l} 
animal \\
inteiro
\end{tabular} & músculo \\
\hline 1.000 & 24 & 2,40 & 396,75 & 15,09 & 3,80 & 0,396 & 0,628 \\
\hline 1.021 & 27 & 2,64 & 264,92 & 16,28 & 6,14 & 0,259 & 0,602 \\
\hline 1.030 & 21 & 2,03 & 268,25 & 8,66 & 3,22 & 0,260 & 0,412 \\
\hline $\begin{array}{l}1.037 \\
\end{array}$ & 25 & 2,41 & 396,74 & 16,43 & 4,14 & 0,382 & 0,657 \\
\hline 1.053 & 16 & 1,51 & 305,82 & 4,75 & 1,55 & 0,290 & 0,296 \\
\hline 1.090 & 28 & 2,56 & 533,44 & 17,33 & 3,24 & 0,489 & 0,618 \\
\hline 1.112 & 25 & 2,24 & 304,46 & 23,57 & 7,74 & 0,273 & 0,942 \\
\hline 1.141 & 28 & 2,45 & 142,94 & 22,22 & 15,57 & 0,125 & 0,793 \\
\hline 1.159 & 25 & 2,19 & 499,68 & 16,05 & 3,21 & 0,431 & 0,642 \\
\hline 1.289 & 25 & 1,93 & 412,71 & 10,57 & 2,56 & 0,320 & 0,422 \\
\hline 1.300 & 20 & 1,53 & 404,11 & 2,51 & 0,62 & 0,310 & 0,125 \\
\hline 1.343 & 33 & 2,45 & 601,68 & 16,43 & 2,73 & 0,448 & 0,497 \\
\hline 1.351 & 26 & 1,92 & 843,96 & 23,26 & 2,75 & 0,624 & 0,894 \\
\hline 1.440 & 22 & 1,52 & 527,35 & 6,21 & 1,17 & 0,366 & 0,282 \\
\hline 1.452 & 37 & 2,54 & 663,19 & 13,22 & 2,00 & 0,456 & 0,360 \\
\hline 1.453 & 40 & 2,75 & 368,56 & 16,87 & 4,57 & 0,253 & 0,421 \\
\hline 1.484 & 33 & 2,22 & 947,46 & 17,21 & 1,81 & 0,638 & 0,521 \\
\hline 1.526 & 23 & 1,5 & 636,95 & 4,75 & 0,74 & 0,417 & 0,206 \\
\hline 1.613 & 32 & 1,98 & 242,03 & 10,57 & 4,36 & 0,150 & 0,330 \\
\hline 1.630 & 25 & 1,53 & 546,53 & 4,22 & 0,77 & 0,335 & 0,211 \\
\hline 1.637 & 31 & 1,89 & 607,62 & 22,71 & 3,73 & 0,371 & 0,732 \\
\hline 1.713 & 25 & 1,45 & 642,13 & 9,97 & 1,55 & 0,374 & 0,398 \\
\hline 1.729 & 31 & 1,75 & 912,51 & 31,53 & 4,91 & 0,515 & 1,017 \\
\hline 1.915 & 27 & 1,40 & 798,59 & 13,28 & 1,66 & 0,417 & 0,491 \\
\hline
\end{tabular}




\begin{tabular}{|c|c|c|c|c|c|c|}
\hline 2.044 & 30 & 1,46 & $\|1112,54\| \quad 15,94$ & 1,43 & $0,550 \|$ & 0,531 \\
\hline$\overline{\mathrm{X}}$ & & 2,01 & & 3,43 & 0,317 & 0,521 \\
\hline d.p. & & 0,44 & & 3,07 & $0,128 \|$ & 0,233 \\
\hline
\end{tabular}

\begin{tabular}{|c|c|}
\hline $\begin{array}{l}\text { Figura 1 . Massa muscular radular média de } \\
\text { Pomacea lineata em relação à massa dos animais } \\
\text { inteiros (alimentados). }\end{array}$ & $\begin{array}{l}\text { Figura 2. Massa muscular radular média de } \\
\text { Pomacea lineata em relação à massa dos animais } \\
\text { inteiros (jejunos). }\end{array}$ \\
\hline $\begin{array}{l}\text { Figura 3. Porcentagem média do consumo } \\
\text { horário de Oxigênio dos músculos radulares de } \\
\text { Pomacea lineata em relação ao consumo horáric } \\
\text { de Oxigênio dos animais inteiros (alimentados). }\end{array}$ & $\begin{array}{l}\text { Figura 4. Porcentagem média do consumo } \\
\text { horário de Oxigênio dos músculos radulares de } \\
\text { Pomacea lineata em relação ao consumo horário } \\
\text { de Oxigênio dos animais inteiros (jejunos). }\end{array}$ \\
\hline
\end{tabular}

\section{REFERÊNCIAS}

ANKEL, W.E. Erwerb und Aufnahme der Nahrung bei den Gastropoden. Zool. Anz. Supl. Leipzig, v.11, p.223-95, 1938.

BYSOVA, J.B. Respiratory metabolism in some millipedes (Diplopoda). Rev. Ecol. Biol. Sol., v. 4, n. 4, p.611-24, 1967. 
CRAVEIRO, J.P.M. Efeito da redução de íons sódio, cálcio e potássio nas contrações espontâneas do esôfago de Pomacea lineata SPIX, 1827 (Mollusco-GastropodeProsobrânquio). Arq.Biol.Tecnol., v.40, n.2, p.369-75, 1997.

CHURCHWELL, M. A comparison of whole animal and summated tissue respiratory rates of the abalone Haliotis cracherodii. Diss. Abstr. Int., v.33B, n.10, p.5064, 1973.

DEYRUP-OLSEN, I. \& MARTIN, A.W. Osmolyti processing in the gut and na important role of the rectum in the land slug, Ariolimax columbianus (Pulmonata, Arionidae). Jour.Exp.Zool. v.243, p.33-8, 1987.

DITAD, A.S.F., MENDES, E.G. \& BIANCONCINI, M.S.C. Influence of body mass and enviroment oxygen tension on the oxygen consumption rates of na enteropneust, Glossobalanus crozieri. Braz. J. Med. Biol. Res., v.30, n.12, 1997.

FÄNGE, R. \& MATTISSON, A. Studies of the physiology of the radula muscle of Buccinum undatum. Acta Zool., Stockholm, v.39, p.53-64, 1958.

GRAHAM, A. Molluscan diets. Proc. Malc. Soc. Londom, v.31, p.144-159, 1955.

GROMYSZ-KALKOWSKA, K. The influence os body weight, external temperature, season of the year and fasting on respiratory metabolism in Polysdesmus complanatus L. (Diplopoda). Folia Biol. (Kracow), v.18, n.4, p.311-26, 1970.

GROMYSZ-KALKOWSKA, K. Some exogenous and endogenous effects on rate of respiration in Strongylosoma pallipedes (Olivier) (Diplopoda) and behavior in temperature gradient. Folia Biol. (Kracow), v.21, n. 3, p.251-69, 1973.

GROMYSZ-KALKOWSKA, K. \& STOJALOWSKA, W. Oxygen consumption level in Glomeris connexa C.L. Koch (Diplopoda) in different physiological states. Folia Biol. (Kracow), v.19, n.4, p.457-72, 1971.

LITTLE, C. Aestivation and ionic regulation in two species de Pomacea (Gastropoda, Prosobranquia). J. exp. Biol., Cambridge, Gt. Brit., v.48, p.569-85, 1968.

MARQUES, M.M. \& BARBOSA, F. Na fauna do fundo, o retrato da degradação. Ciência Hoje, v.30, n.175, p.72-5, 2001.

MELO, L.E.L. de. O uso do gastrópode Pomacea lineata (SPIX, 1827) como indicador de toxidade em mananciais de água doce no Nordeste do Brasil: Uma proposta metodológica. 2000. Resumo. (Dissertação). PRODEMA. Programa Regional de Pós-Graduação em Desenvolvimento e Meio Ambiente - Universidade Federal da Paraíba. Disponível em http://gw- 
prpg.prpg.ufpb.br/ prodema/dissertações/por_ano.htm Acesso em: 20/09/2002.

MENDEL, L.B. \& BRADLEY, H.C. Experimental studies on the physiology of the molluses. Am. J. Physiol., Boston, v.13, p.17-29, 1905.

MANWELL, C. The chemistry and biology of hemoglobin in some marine clams. I. Distribution of the pigment and properties of the oxygen equilibrium. Comp. Biochem. Physiol., New York, v.8, p.209-18, 1963.

OWEN, G. FEEDING. In: WILBUR, K.M. \& YONGE, C.M. Physiology of Mollusca. Vol. 2. New York. Acad. Press., p.1-51, 1966.

SANTOS, C.A.Z. \& MENDES, E.G. Oxygen consumption of the amphibious snail Pomacea lineata: Influence of weight, Sex and environments. Comp. Biochem. Physiol., New York, v.69 A, p.595-98, 1981.

SANTOS, C.A.Z., PENTEADO, C.H.S. \& MENDES, E.G. The respiratory responses of na amphibious snail, Pomacea lineata (Spix, 1827) to temperature and oxygen tension variations. Comp. Biochem. Physiol., v.86A, p.409-15, 1987.

SILVA, R.S. da, PENTEADO, C.H.S. \& BOCCARDO, L. Relações fisioecológicas no diplópodo Oxidus gracilis (Polydesmida, Strongylosomidade): $O$ consumo de oxigênio em relação ao tamanho, sexo e condição alimentar. HOLOS Environment, v.1, n.1, p. 67-82, 2001.

VERNBERG, F.J. Study of the oxygen consumption of excised tissues of certain marine decapod crustacea in relation to habitat. Physiol. Zool., Chicago, v.29, p.227-34, 1956.

WITTENBERG, B.J. Myoglobin facilitated oxygen diffusion: role of myoglobin in oxygen entry into muscle. Physiol. Ver. Baltimore, v.50, p.550-635, 1970. 